\title{
CARACTERIZACIÓN DE LOS ASPECTOS BIOÉTICOS EN LOS RECLAMOS REALIZADOS ANTE LA SUPERINTENDENCIA DE SALUD DE CHILE EN EL ÁREA DE ODONTOLOGÍA
}

\author{
Francisco José Portela León ${ }^{1}$
}

Resumen: La presente investigación identifica los denominadores comunes de los reclamos presentados ante la Superintendencia de Salud de Chile en el Formulario de Solicitud de Mediación a prestadores privados en el área de odontología, que evidencian el quiebre de la relación odontólogo-paciente, y, adicionalmente, analiza el contenido de las narraciones que expresan la insatisfacción por expectativas no cumplidas en la atención clínica, estableciendo su componente ético. Consta de un análisis cualitativo de las narraciones realizadas por los pacientes en los formularios de solicitud de mediación, utilizando la técnica de análisis de contenido para la exploración categórica y un análisis cuantitativo que aportó datos numéricos sobre las especialidades odontológicas involucradas y datos sociodemográficos. Una parte de las insatisfacciones presentadas están en concordancia con el poco manejo ético por parte de los profesionales, lo cual deteriora la relación interpersonal. Los reclamos podrían disminuir con la inclusión de pautas éticas en la relación odontólogo-paciente, apuntando a un reconocimiento del otro, su dignidad y a la constante mejora de nuestra conciencia ética como profesionales.

Palabras clave: bioética, reclamos, odontología, relación clínica

\section{Characterization of bioethical aspects of complaints to the Health Authority in Chile in the odontological area}

\begin{abstract}
This investigation identifies common denominators of complaints presented to Health Superintendent in the form devoted to mediation related to private providers, evincing the breakdown of the odontologist-patient relationship and in addition analyzes the contents of narratives expressing dissatisfaction with unfulfilled expectations, emphasizing its ethical component. There is a qualitative analysis of narratives included in the forms using content analytic techniques and a quantitative analysis providing data on odontological specialties involved and sociodemographic data. Part of the dissatisfaction is related to bad ethical handling on the part of the professionals, deteriorating the personal relationship. Complaints could be reduced by the inclusion of ethical norms in the interpersonal relation, pointing towards recognition of the other, his/her dignity and the improvement of ethical consciousness as professionals.
\end{abstract}

Key words: bioethics, complaints, odontology, clinical relationship

\section{Caracterizaçáo dos aspectos bioéticos das reclamaçóes realizadas perante a Superintendência de Saúde do Chile na área de odontologia}

Resumo: A presente investigação identifica os denominadores comuns das reclamaçốes apresentadas perante a Superintendência de Saúde do Chile no Formulário de Solicitação de Mediação a prestadores privados na área de odontologia, que evidenciam a quebra da relação odontólogo-paciente, e, adicionalmente, analisa o conteúdo das narrativas que expressam a insatisfaçáo por expectativas não cumpridas na atenção clínica, estabelecendo seu componente ético. Consta de uma análise qualitativa das narrativas realizadas pelos pacientes nos formulários de solicitação de mediação, utilizando a técnica de análise de conteúdo para a exploração categórica e uma análise quantitativa que trouxe dados numéricos sobre as especialidades odontológicas envolvidas e dados sociodemográficos. Uma parte das insatisfaçóes apresentadas estáo em concordância com o pouco manejo ético por parte dos profissionais, o que deteriora a relação interpessoal. As reclamaçóes poderiam diminuir com a inclusão de pautas éticas na relação odontólogo-paciente, apontando para um reconhecimento do outro, sua dignidade e a constante melhora de nossa consciência ética como profissionais.

Palavras-chave: bioética, reclamações, odontologia, relação clínica

\footnotetext{
${ }^{1}$ Odontólogo, Magíster en Bioética, Universidad de Chile, Chile. Correspondencia: franciscoportela@hotmail.com
} 


\section{Introducción}

Un Estado democrático supone que el ciudadano tiene el derecho a ser escuchado. El privilegio de la petición es inherente a la ciudadanía libre. La Superintendencia de Salud de Chile, en 2005, desarrolló una nueva modalidad para solucionar los conflictos con prestadores de salud privados y estableció un método previo a la instancia judicial llamado "mediación". Se ha abandonado la judicialización directa como procedimiento para solucionar los conflictos entre el profesional de salud y los pacientes. En la mediación no se dictaminan sentencias judiciales, sino que se promueve el diálogo personal entre las partes. Solo lleva en funcionamiento pocos ańos y el número de ingresos se ha incrementado gradualmente. A través de su mirada, los mismos pacientes, o sus representantes, realizan su solicitud mediante un formulario en el que narran su insatisfacción en una intervención clínica.

Los reclamos en odontología son un fenómeno social determinado por múltiples factores, pero su mayor causa es una relación odontólogo-paciente defectuosa y, particularmente, por la falta de la atención a preceptos éticos en su práctica clíni$\mathrm{ca}(1)$. Los reclamos no representan únicamente negligencias o errores clínicos o técnicos; existen otros problemas en la atención odontológica y es fundamental comprender qué reclaman los pacientes y actuar sobre dichas causas. El respeto y la dignidad conforman el requerimiento de los pacientes en la mayoría de los reclamos(2). La importancia de estudiar y analizar los reclamos es determinante para identificar y aprender de las falencias en la relación odontólogo-paciente y así establecer las acciones que determinen una mejoría de la misma. Los reclamos nos invitan a investigar el modo en que los dilemas éticos son percibidos y construidos por aquellos a quienes afectan y cómo estos pacientes manejan y narran tales conflictos, en un mundo crecientemente cambiante y diverso. Así, una mirada más contextual y situacional entrega una mejor respuesta a las dimensiones empíricas de los problemas morales(3). La relación clínica no se basa exclusivamente en el buen desempeño profesional del odontólogo en su parte técnica, existen muchas más competencias éticas que generan relaciones basadas en la confianza y el respeto a la dignidad del paciente.

La buena comunicación en la relación clínica es imprescindible para entablar un acercamiento con nuestros pacientes, logrando un adecuado objetivo terapéutico y la satisfacción los mismos. Es un requisito ético que se involucra en el desarrollo de la confianza, en la transmisión de información y el manejo de expectativas, y es la base de un proceso de consentimiento informado, el que, además de ser un requisito legal, legitima y fundamenta nuestra práctica clínica.

El sistema de registro de reclamos de la Superintendencia de Salud es una fuente para la recolección de insatisfacciones narradas desde el punto de vista de los pacientes. Su caracterización servirá para entender la insatisfacción e identificar su componente ético. Además, muestra datos estadísticos y sociodemográficos que sirven para realizar recomendaciones encaminadas a promover la mejora de la práctica ético-profesional en odontología.

\section{Reclamos en odontología}

Actualmente, los pacientes se presentan como consumidores, como usuarios de servicios y como participantes activos del proceso de atención. El paciente es crítico y busca ofertas, compara los paquetes de servicios y escoge a su odontólogo por diversas características. Además, tiene mayor conciencia de sus derechos y se organiza en grupos, lo que aumenta las demandas y reclamaciones(4). La principal protección contra los reclamos de los pacientes es una buena relación clínica, apoyada en la sinceridad, la objetividad y la confianza. Cuando la relación clínica es buena, los reclamos son infrecuentes, inclusive en casos de negligencia; cuando es mala, los reclamos se multiplican, incluso sin fundamento(5). Existe un creciente aumento de demandas médico-legales que involucran a profesionales, las que se atribuyen, en parte, el encarecimiento de la atención y a la aparición de la llamada "medicina defensiva", es decir, decisiones médicas adoptadas más por temor a los reclamos o solicitudes de los pacientes que 
por motivos clínicos. Entre estas medidas, algunas se consideran negativas (por ejemplo, pedir exámenes en exceso) y otras positivas (dar mejor trato al paciente)(6).

Las reacciones de los odontólogos hacia la amenaza de posibles reclamos se orientan hacia aspectos técnicos de la atención(2) y no hacia el fortalecimiento en aspectos éticos en la relación clínica. Los problemas ocultos de la relación odontólogopaciente son el origen de las características conflictivas del vínculo entre las dos partes, resaltando siempre la falta de claridad de la relación propiamente dicha, como en las intervenciones o procedimientos a ejecutar. Los estudios muestran que en más del $90 \%$ de cualquier reclamo o demanda se encuentra una relación medico-paciente defectuosa, dando lugar a que un resultado clínico no deseado sea interpretado como falla o error, incapacidad del profesional y cualquier otra peculiaridad negativa origina una denuncia, presuponiendo mala práctica(7).

El objetivo deseable no es reaccionar respondiendo las demandas y reclamos médicos, sino usar todos los medios para prevenirlos, analizándolos como una estrategia de mejoramiento continuo de la calidad de la atención. Existe un crecimiento de exigencias y expectativas generales de los pacientes, que no deben manejarse solo solucionando y satisfaciendo los reclamos personales, porque hay riesgo de un círculo vicioso que no permite profundizar en los motivos (de pacientes y odontólogos) que subyacen a la manifestación de inconformidad, por lo que es importante identificar, comprender y solucionar las anomalías de fondo de los reclamos(2).

Método

La presenta investigación utiliza los enfoques cualitativo y cuantitativo de forma complementaria. Para el estudio cualitativo se utiliza el análisis de contenido de las narraciones de los pacientes en los formularios llenados ante la Superintendencia de Salud, considerando los ítems en la parte de la descripción del problema y la solicitud al prestador. Además, se realiza un análisis cuantitativo de tipo descriptivo de datos sociodemográficos y especialidades involucradas reveladas en el formulario.
Se estudian los 156 formularios ingresados a la Superintendencia de Salud del área de odontología a prestadores privados en el periodo comprendido entre 2005 y 2010, analizando todos los ítems, exceptuando la información referida a la identidad del individuo (nombre, R.U.T, teléfono y/o e-mail), y también el nombre de los mediadores seleccionados.

\section{Método cuantitativo}

Se efectuó un estudio descriptivo de corte transversal, a partir de los reclamos recibidos en la Superintendencia de Salud y registrados en el formulario "Solicitud de Mediación con Prestadores Privados". Se analizó la información en el formulario de las siguientes variables: número de ingreso, fecha de ingreso, fecha de los hechos, edad, asegurador (Isapre, Fonasa u Otro), sexo, relación de la persona que presenta el reclamo en caso de no ser la persona dańada (Familiar u Otro), representante (Abogado, Familiar u Otro), prestador reclamado (Institucional o Persona Natural), especialidad, comuna y región. Para el análisis se empleó estadística descriptiva. Para este propósito se utilizó el paquete estadístico Epi-info $0^{\circ}$ versión 3.5.1. A las variables se les asignaron códigos numéricos, constituyendo una base de datos, las cuales fueron objeto de análisis de frecuencias.

\section{Método cualitativo}

El análisis cualitativo se basó en un análisis de contenido. Esta técnica buscó contenidos manifiestos y latentes. Posteriormente, aquella información cuantificable fue codificada de acuerdo con las categorías expresadas en investigaciones internacionales (CONAMED). Se conformó entonces una matriz que agrupó cada una de las categorías de acuerdo con el número asignado por la Superintendencia. Se realizó un análisis por caso ingresado y luego por la caracterización de los problemas, identificando los componentes comunes en los relatos. Se utilizó la metodología documental, basada en análisis de narraciones según los criterios de inclusión. Se regresó al material original cuantas veces fue necesario, en un proceso reiterado entre el material original, las ideas y conceptos. La revisión sistemática se detuvo 
cuando se consideró haber obtenido la saturación teórica posible mediante los formularios.

\section{Resultados}

\section{Características de la población estudiada}

El gráfico 1 muestra un aumento significativo de los reclamos en el transcurso del periodo estudiado. De acuerdo con el género, el 68\% de los reclamos correspondieron al sexo femenino y el $32 \%$ al sexo masculino.

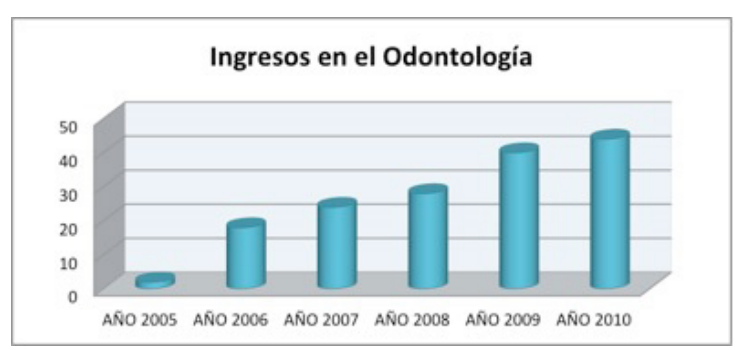

Gráfico 1

Según la distribución de la edad de los pacientes, el primer lugar fue para el grupo de 46-60 años con 34 casos (22\%); el segundo para el de $31-45$ años con 33 casos (21\%) y el tercero para el de 15-30 años con 23 casos (15\%).

En cuanto a la persona que presenta el reclamo, los mismos afectados fueron el mayor porcentaje con el 46\%, seguido de los abogados, con un $20 \%$, y por último el grupo en el que el reclamo fue realizado por un familiar, con un 15\%.

Caracteristicas de las instituciones aseguradoras y personal involucrado en el reclamo

Respecto de las personas reclamadas: el odontólogo con 45 casos (29\%); la institución y el odontólogo con 41 casos (26\%) y solamente la institución con 35 casos (22\%).

En relación con el tipo de institución aseguradora: Fonasa con 59 casos (38\%), Isapres con 40 casos $(25 \%)$ y Otros con 9 casos $(6 \%)$.

\section{Caracteristicas demográficas}

El formulario permite una representatividad a escala nacional y posibilita la comparación entre categorías sociales, entre especialidades involucradas, entre diferentes regiones del país y entre las distintas comunas de la Región Metropolitana.

Por Región, la mayor parte de los reclamos corresponden a la Metropolitana, con 81 casos (52\%), el segundo para la VIII Región, con 19 casos (12\%), el tercero para la IV Región con 13 casos (8\%), y los casos restantes corresponden a las demás regiones y se muestran en el gráfico 2 . En los gráficos 3 y 4 se muestra la tasa en relación con el número de habitantes por región y comunas de la Región Metropolitana (de acuerdo con el Instituto Nacional de Estadísticas del XVII Censo Nacional de Población, realizado en abril de 2002).

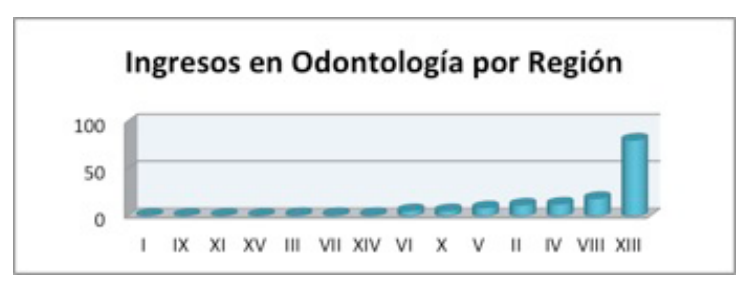

Gráfico 2

Las cinco primeras regiones con mayor tasa de reclamos por número de habitantes, de mayor a menor, fueron: II Región de Antofagasta, IV Región de Coquimbo, XIII Región Metropolitana, XI Región de Aisén y VIII Región de Biobío.

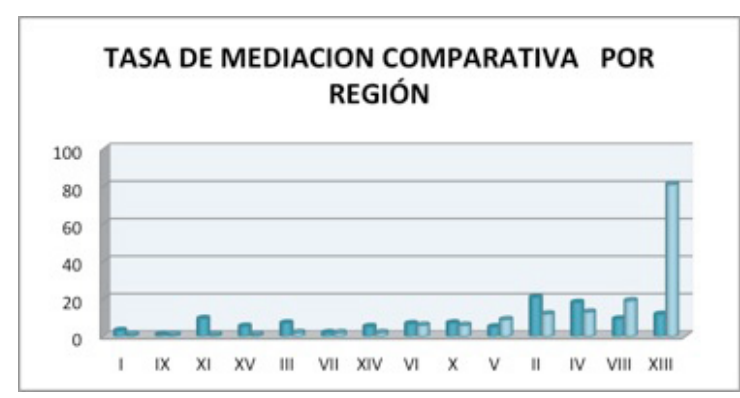

Gráfico 3

En cuanto a la tasa de reclamos por comunas en la Región Metropolitana, Santiago es la comuna con una mayor tasa de reclamos, seguida de Las 
Condes, Quinta Normal, Providencia, Quilicura, Huechuraba y San Miguel.

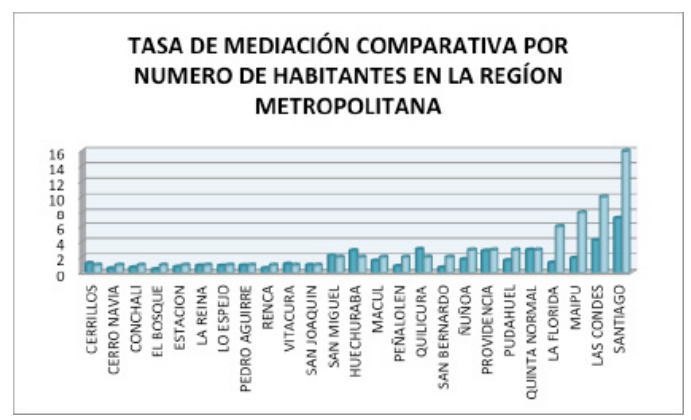

Gráfico 4

\section{Especialidades Odontológicas}

Las especialidades odontológicas, en orden decreciente, fueron endodoncia $(17,9 \%)$, prótesis fija $(13,5 \%)$, exodoncia $(10,9 \%)$, implantes $(10,9 \%)$, prótesis removible $(7,1 \%)$, restauraciones $(5,8 \%)$, ortodoncia $(4,5 \%)$, periodoncia $(1,3 \%)$, estética $(1,3 \%)$, expresadas en gráfico 5 .

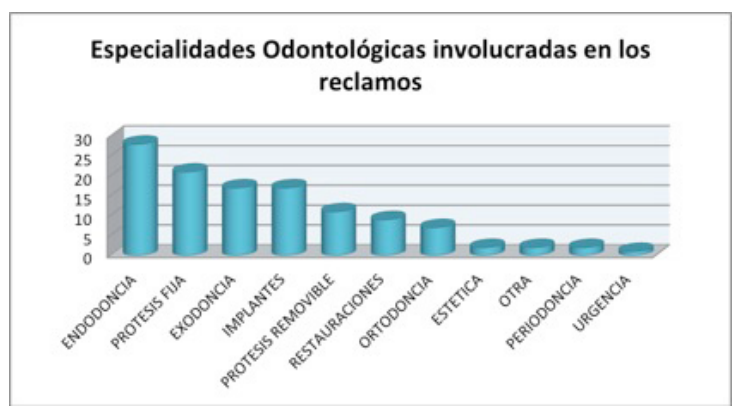

Gráfico 5

Pretensiones de los reclamos

Los reclamos tienen como principal solicitud una pretensión económica que se evidencia en un 35\% de los casos que representan una indemnización; simultánea o independientemente manifiestan otros tipos de pretensión, como la devolución del dinero, en un $23 \%$, un nuevo trabajo con otro profesional, $8 \%$, un nuevo tratamiento o término con el mismo profesional, $5 \%$, entre otras que se muestran en el gráfico 6.

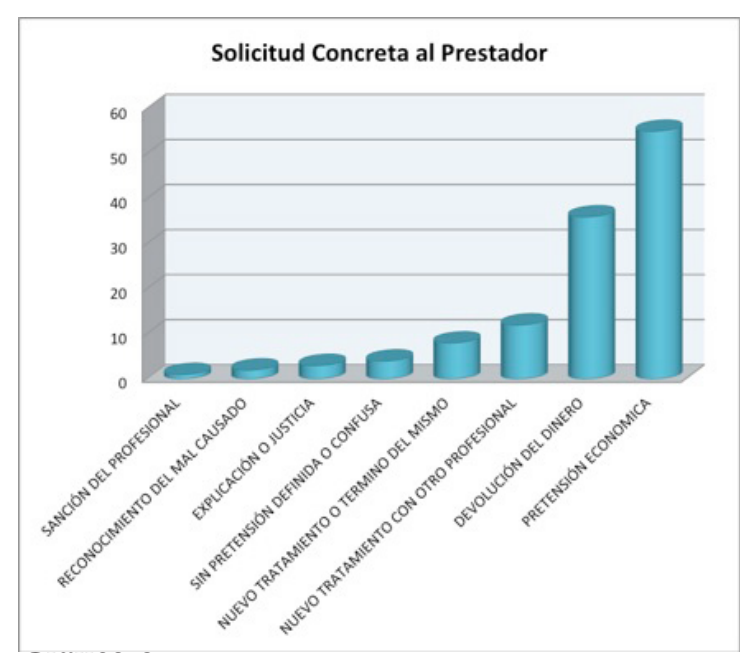

Análisis de las narraciones

Los problemas descritos en los reclamos exhibieron muchas similitudes con las categorías expresadas en otras investigaciones(8). La principal categoría expuesta fue la de problemas de comunicación, seguida por la generación de falsas expectativas, ética profesional deficiente, comentarios sobre otro profesional, técnicas rehabilitatorias mutilantes e incumplimiento de obligaciones.

La comunicación, como eje central en la relación odontólogo-paciente, es uno de los mayores desencadenantes de conflictos en las narraciones de los pacientes. La calidad de la relación interpersonal y los problemas de comunicación son frecuentemente activadores de reclamos. Los siguientes ejemplos son representativos.

(...) En una primera instancia comenzó este tratamiento la dentista —. la cual, luego de sufrir un accidente lo continuó el dentista $\longrightarrow$ el cual hizo, dentro del tratamiento, una pieza que no correspondía al presupuesto, luego de éste se me quiso obligar a pagar ésta, a lo cual me negué, ya que nunca nadie me consultó al respecto, por lo cual posteriormente se me negó la posibilidad de continuar con mi tratamiento cancelado de forma anticipada, dejando tres piezas sin terminar el tratamiento (...) (104)2.

\footnotetext{
${ }^{2}$ Los números entre paréntesis en estos párrafos testimoniales, corresponden a la correlación interna del reclamo que se cita en cada caso. Se conserva el anonimato de las personas involucradas, por motivos éticos.
} 
Reclamos realizados ante la Superintendencia de Salud de Chile en el área de odontología - Francisco Portela

(...) El doctor... no respondió mis preguntas..., le volvi a preguntar ¿Qué tiene mi hija? ¿Debo pedir hora con la endodoncista? No respondió y entró a su consulta. La secretaria me pidió que abonara al tratamiento, ¿Qué tratamiento? Le dije, solo solicité una evaluación y radiografía, cancelé solo lo solicitado. Lo único que se le solicitó y debió hacer era indicarme su apreciación diagnóstica y derivarme donde la especialista que correspondia (...) (114).

Los reclamos presentan múltiples modalidades de problemas comunicacionales que generan un trato percibido como inadecuado, de maltrato, poco respeto a los pacientes como personas, insuficiente consideración y ausencia de sensibilidad ante su realidad.

(...) Me propuso hacerme él un implante por ser mucho más cómodo que una prótesis tradicional. Me dijo que lo pensara, dándome el valor del implante (incluso me mostró una prótesis de otro paciente para que viera cómo me podía quedar). Lo pensé y decidi hacerme el implante y el doctor __. me dijo que no demoraba más de 20 días. Me citó el miércoles su consulta para operarme y en ese momento me dice que no me operaría ahi y me llevó a una clinica a dos cuadras de su consulta y me dijo que me operaria su colega el doctor_. Terminada la operación, me mandó de vuelta a su consulta de a pie, con todo mi dolor, caminando de nuevo las 2 cuadras para llegar a su consulta. Me siguió controlando el doctor hasta mayo, siendo que el me habia dicho que el tratamiento duraba 20 días. Volví a mi casa desesperada por mi situación, al ver que tenía que esperar tanto tiempo para mis dientes. Después me llamó la secretaria del doctor __ para que volviera a su consulta, yo le dije que no quería volver, ya que consideraba que era una falta de respeto que me hubiera paseado por la calle con todo mi dolor. Me decidi volver a la consulta y empezó a hacerme prótesis provisorias y yo le rogaba que me pusiera dientes chicos que se parecieran a los mios, me mostraba dientes para que viera el tamaño yo le decía que si me gustaban pero me ponía otros de tamaño más grandes y asi fue pasando el tiempo y yo siempre le reclamé su trabajo, ya que me encontraba desconforme. Después de varias prótesis provisorias recién en me terminó el trabajo. Me vine a mi casa muy molesta, ya que para mi el trabajo que me hizo no era lo que me prometió (...) (115).

(...) El jueves 21 de diciembre le llamo a 1a hora, contesta la secretaria___ me dice que está ocupado que llame después, lo llame 2-3-4 veces y era la misma respuesta, que no me podia atender, hasta que mi hijo lo llamó de su celular y pidió enérgicamente hablar con él, le contestó y le dijo que me atendería después de las fiestas de fin de año, o sea para enero. El mismo jueves 21, tipo 17 horas, fui a una clínica que hay al frente de mi casa a consultar por el dolor, me examinaron y me dicen que la raiz esta fracturada y que hay que extraer la pieza. Le llamo directamente de la clinica para informarle lo que él ya sabría, motivo por el cual él no me quería atender, me contesta la secretaria y me dice: un momento voy a consultarle y le dice que me diga que ya se retiró (lo escucho yo a través del teléfono) (...) A mediados de marzo fui personalmente y me hice acompañar de mi hijo, nos hizo esperar pero nos tuvo que atender y me dijo lo mismo, que las radiografías y todo lo demás él no me los devolvía, porque eran de su propiedad. Yo le contesté que reclamaría contra él y me dijo: me da lo mismo, soy amigo de grandes jueces y abogados, asi que haga lo que quiera: yo tengo quien me defienda (...) (42).

Con frecuencia, los reclamos ante la Superintendencia de Salud constituyen la última instancia a la que recurren los pacientes. La falta de respuesta adecuada en los mismos servicios de salud provoca que los pacientes busquen resolver su problema fuera de la institución, como se observa en los siguientes ejemplos:

(...) Ese día hablé con una enfermera jefa, que fue la única que dio la cara. Volvi a la semana siguiente, puse una queja, luego de semanas puse otra y nunca pasó nada, me cansé de ir todos los dias a perder tiempo. Mientras me hacia el tratamiento, se negaba a informarme lo que hacia en la muela y me hizo doler mucho (...) (45).

(...) Mi padre reclamó ante diferentes instancias, hablando con el doctor jefe, el señor quien respondió vía mail el $\longrightarrow$ manifestando que está "a la espera de una resolución por parte de la gerencia técnica y comercial". Nunca en- 
tregó solución alguna hasta hoy, lo que ha motivado este reclamo (...) (33).

Algunos reclamos muestran una expectativa que no fue cumplida, a los ojos de los pacientes, en algunos ambientes y contextos determinados, lo que se ilustra en las siguientes situaciones:

(...) Un dia, sin avisarme previamente, procede a cortarme las dos piezas dentales superiores delanteras (8 y 9) sin mi consentimiento, argumentando que "no tenían salvación" y que "solo las raices sirven", y señalándome que en su lugar pondria coronas dentales que se verian mucho más hermosas que mis dientes propios. Luego me pasa un espejo para que me vea y yo, al verme, caigo en una profunda amargura al verme asi, y él me señala que mientras tanto pondría coronas provisorias y que en unas sesiones más la cambiaría por las definitivas que serian de porcelana (...) (132).

(...) Yo pensando que iban a pagar un pabellón, operaron a mi hija en su consulta colocándole la anestesia general y operándola en la silla que revisa a los pacientes, el me habia dicho que la anestesia duraba $30 \mathrm{~min}$ y la operación la iba hacer en 20. Pasaron las horas y la anestesia duro 4 horas. Mi señora averigua que el doctor no tiene permiso para operar y menos con anestesia general (...) (119).

Las expectativas no cumplidas, en cuanto al tiempo de tratamiento propuesto por el odontólogo y la durabilidad en el tiempo de los mismos, son una variable presente en muchos relatos, por ejemplo:

(...) Se me indicó que el tratamiento completo demoraría máximo 6 meses y se me exigió el pago total por adelantado y al contado. Así lo realicé; sin embargo, no recibi ningún comprobante de pago ni menos una boleta. Llevo un año ocho meses asistiendo a la consulta del señor _. Durante este periodo en reiteradas oportunidades se me ha cambiado la hora de atención a último momento y muchas veces sin siquiera avisarme, situación por la cual he llegado a atenderme y simplemente no se me atiende. Se ha abusado de mi paciencia, se ha hecho caso omiso de mis reclamaciones, se ha perjudicado mi calidad de vida, y después de un año ocho meses el tratamiento ofrecido no ha terminado (...) (131).

Algunos reclamos ponen de manifiesto irregularidades que, por sí solas, no motivaron entablar el reclamo, pero son mencionadas para justificarlo y las pretensiones que solicitan.

(...) Según él, era docente de la (...) Hasta el 6 de abril de __ Llama para no atenderme más sin explicación ni razón alguna. El día 17 de abril ___ fui a la facultad en la cual me dijeron que nunca debia haber salido de ésta, pero él nos llevó engañados, y que él era un alumno y no docente, pero él lo hizo en forma particular y no por intermedio (...) (44).

Algunos pacientes ven como, en ciertas ocasiones, se ven mutilados de su corporeidad y lo manifiestan en los reclamos.

(...) Me tapó 2 muelas y me mandó a hacer una prótesis, lo cual la prótesis me quedó mal hecha y la doctora optó por extraérmelas antes de hacer un tratamiento o buscar otra alternativa, se fue a lo más fácil (...) (129).

(...) ___ fue llevada al consultorio, siendo atendida por el doctor__ quien le habría extraido una pieza dental definitiva sin tomar antes una radiografía, perdiendo asi su diente (...) (21).

Existe una responsabilidad administrativa involucrada en los motivos para interponer un reclamo, estos son incumplimientos de obligaciones que no se refieren al ejercicio profesional, sino a la no prestación del servicio como tal, por ejemplo:

(...) Me informan que la administración anterior utilizó mal los fondos y que, por lo tanto, tengo que volver a pagar lo que me falta del tratamiento (...) (19).

(...) Fui al edificio a hablar con el dentista, el cual me dijo que el ya no trabajaba para la empresa y que también ya no existía mas la clínica (...) (137). 


\section{Discusión}

La bioética contribuye al reconocimiento de indicadores que ayudan a identificar los cambios que la práctica y la educación odontológica requieren. Las actividades orientadas hacia la búsqueda de estrategias para preservar la dignidad de los pacientes son fundamentales. La atención de reclamos constituye uno de los mecanismos del sistema de salud para reconocer la dignidad de los pacientes. El Formulario de Solicitud de Mediación de la Superintendencia de Salud recoge la percepción de los pacientes acerca de la satisfacción en la relación clínica, y es un instrumento que amerita un abordaje metodológico mixto, para evidenciar aspectos éticos involucrados en la atención. Los datos numéricos nos aportan un contexto, profundizado por las particularidades narradas por los pacientes, dando un mapa más claro de las variables que pueden incorporarse en la relación odontólogo-paciente. El reclamo aporta una visión, en ocasiones, oculta por la perspectiva meramente clínica de los odontólogos al enfrentar los dilemas éticos que involucra la práctica clínica.

La insatisfacción no siempre es sinónimo de reclamo. Probablemente han quedado excluidos algunos conflictos éticos que no llegaron a la instancia de la mediación, por múltiples factores: entre otros, el tiempo para generar el mismo, los costos, la posibilidad de no llegar a probar un daño, el enfrentamiento con el personal de salud, la estigmatización, etc. De modo que es probable que estos resultados no reflejen toda la magnitud del problema. El estudio de los reclamos de los pacientes es un recurso para la bioética, teniendo en cuenta que de todas las no conformidades se puede aprender.

Existe un aumento gradual y significativo del número de reclamos en odontología desde que se inicio el sistema de mediación de la Superintendencia de Salud y va de la mano con la confianza que pueda tener la sociedad, así como con elementos y formas diferentes de manifestar opiniones. El modelo de la mediación es utilizado en otras instituciones del Estado para promover, por medio del diálogo, una vía resolutiva del conflicto, como alternativa a la judicial.
En cuanto a la diferencia por género, se observa una marcada tendencia a mayores ingresos de reclamos de mujeres; además, los rangos etarios mayoritarios tienen valores muy similares a estudios realizados por otros autores(8), y coinciden también con el uso de los servicios odontológicos mayoritariamente por las mujeres y en estas edades(9). Algunos también han investigado la proporción de reclamos interpuestos directamente por los pacientes y por sus representantes; al respecto, en Australia hallaron que un $44 \%$ de los reclamos fue interpuesto por un representante, en Inglaterra casi la mitad fue interpuesta por el mismo paciente(1). Los resultados aquí obtenidos muestran las mismas tendencias con un $46 \%$ de reclamos presentados por el mismo paciente.

Las especialidades odontológicas involucradas en los reclamos difieren de lo encontrado en otros estudios; por ejemplo, en México el mayor número de ingresos lo presenta ortodoncia y en tercer lugar se muestra endodoncia, a diferencia de Chile, donde se muestra endodoncia en primer lugar y ortodoncia es relegada a un séptimo lugar.

Lo que piden los pacientes para resolver el conflicto por lo general es una pretensión económica; sin embargo, hay que destacar los demás factores manifestados en los reclamos, como que el mismo odontólogo termine el tratamiento que está realizando, mostrando una plena confianza en su parte técnica, pero insatisfacción con otros aspectos. Algunos solicitan una explicación, un reconocimiento del mal causado o de lo sucedido, y en ciertos casos una sanción para los odontólogos. En esta investigación, como en otras, existe un porcentaje de pacientes sin una pretensión clara o bien definida, casos que se podrían catalogar como de pacientes que "no piden nada", que sólo buscan narrar una situación, ser escuchados y plantean el reclamo "como fin en sí mismo"(2).

La comunicación y las habilidades interpersonales son una parte fundamental en nuestra práctica clínica odontológica y el carácter ético de la misma, ya que son las que permiten la realización de tareas especificas y conductuales, tales como la realización de un proceso de consentimiento, una historia clínica adecuada, la explicación del 
diagnóstico y el pronóstico, dar instrucciones terapéuticas y asesoramiento de nuestros pacientes. Esta interrelación de habilidades nos permite tener un efecto en la otra persona y dar alivio de la ansiedad o el establecimiento de una relación de confianza(10). La mayoría de los reclamos sobre los médicos están relacionados con temas de competencia comunicativa clínica. Los pacientes quieren médicos con habilidades para diagnosticar y tratar sus enfermedades, así como comunicarse con ellos con eficacia(11).

\section{Conclusiones}

Los reclamos presentados ante la Superintendencia de Salud evidencian diferentes aspectos clínicos y de relación humana. Una parte de las insatisfacciones presentadas están en concordancia con el poco manejo ético por parte de los profesionales, lo cual deteriora la relación interpersonal, por ejemplo, una mala comunicación con los pacientes durante los tratamientos. Los reclamos podrían disminuir con la inclusión de pautas éticas en la relación odontólogo-paciente, apuntando a un reconocimiento del otro, su dignidad y a la constante mejora de nuestra conciencia ética y moral como profesionales.
Los reclamos no deben constituir un sistema aislado de nuestra práctica profesional, tampoco debe ignorarse el modo como el paciente percibió y vivió su atención clínica, ni lo que significa el reclamo para él. Centrarse solo en buscar un error técnico y no un problema en las relaciones personales sería ir en contra de un mejoramiento en la calidad de la atención odontológica y se desconectaría el sistema con la retroalimentación social, fundamental para su evolución.

La educación en valores éticos y la difusión de los mismos en el seno de la profesión odontológica es primordial para realizar un cambio de mentalidad en una vivencia ética de la práctica clínica odontológica y en el mejoramiento de la calidad en la atención centrada en los pacientes.

\section{Agradecimientos}

Profesora María Angélica Sotomayor, Universidad de Chile; profesora Nina Horwitz Campos, presidenta Comité del Programa de Magíster en Bioética de la Facultad de Medicina, Universidad de Chile; Dra. Ana María Fernández Pinto, jefa Sub Departamento de Gestión y Análisis de Reclamos, Superintendencia de Salud, Gobierno de Chile. 
Reclamos realizados ante la Superintendencia de Salud de Chile en el área de odontología - Francisco Portela

\section{Referencias}

1. Triana J. La ética: un problema para el odontólogo. Acta Bioethica 2006; 12(1): 75-80.

2. Infante C. Quejas Médicas. La insatisfacción de los pacientes con respecto a la calidad de la atención médica. México D.F.: Editores de textos Mexicanos; 2006.

3. Horwitz N, Norero C. ¿Por qué investigar en bioética? Rev méd Chile 2002; 130(8): 911-915.

4. Lifschitz A. Comunicación con el paciente difícil. Memoria del VII Simposio Internacional Conamed. Revista CONAMED 2002; 7(3): 18-21.

5. Bertrán J, Collazo E, Gérvas J, et al. Intimidad, confidencialidad y secreto. Guías de ética en la práctica médica. Madrid: Fundación de Ciencias de la Salud; 2005.

6. Ministerio de Salud. Pauta para la elaboración, aplicación y evaluación de guías de práctica clínica. Santiago de Chile: Gobierno de Chile; 2002.

7. Manuell G. Tópicos selectos en la relación médico-paciente. Memoria del VII Simposio Internacional Conamed. Revista CONAMED 2002; 7(3): 14-15.

8. González S, Rivera A, Tena C, et al. Recomendaciones para mejorar la práctica odontológica. Revista AMD LXI 2004; 61(3): 109-116.

9. Consejo Dentistas. Encuesta Poblacional: La salud bucodental en España, 2010. Madrid: Lacer: 2011.

10. Duffy FD, Gordon GH, Whelan G, et al. Assessing competence in comunication and interpersonal skills: The Kalamazzo II Report. Academic Medicine 2004; 79: 495-507.

11. Fong Ha J, Longnecker N. Doctor-Patient Communication: A Review. The Ochsner Journal 2010; 10(1): $38-43$.

Recibido: 10 de noviembre de 2011

Aceptado: 15 de enero de 2012 